\title{
Spin infection enables efficient gene delivery to muscle stem cells
}

\author{
Yusaku Kodaka ${ }^{1,2,3}$, Yoko Asakura',2,3, and Atsushi Asakura1,2,3 \\ ${ }^{1}$ Stem Cell Institute, ${ }^{2}$ Paul and Sheila Wellstone Muscular Dystrophy Center, and ${ }^{3}$ Department of \\ Neurology, University of Minnesota Medical School, Minneapolis, MN
}

BioTechniques 62:72-76 (August 2017) doi 10.2144/000114576

Keywords: muscle stem cell; satellite cell; skeletal muscle; regeneration; retroviral vector

Supplementary material for this article is available at www.BioTechniques.com/article/114576.

Viral vector-mediated foreign gene expression in cultured cells has been extensively used in stem cell studies to explore gene function. However, it is difficult to obtain high-quality stem cells and primary cells after viral vector infection. Here, we describe a new protocol for high-efficiency retroviral infection of primary muscle stem cell (satellite cell) cultures. We compared multiple commercially available transfection reagents to determine which was optimal for retroviral infections of primary myoblasts. Centrifugation force was also tested, and a spin infection protocol with centrifugation at $2800 \times g$ for 90 min had the highest infection efficiency for primary myoblasts. We confirmed that infected muscle stem cells maintain cell proliferation and the capacity for in vitro and in vivo myogenic differentiation. Our new, efficient retroviral infection protocol for muscle stem cells can be applied to molecular biology experiments as well as translational studies.

Skeletal muscle regeneration is mediated by muscle stem cells called satellite cells (1), which are normally mitotically quiescent in adult muscle. After muscle injury or exercise, quiescent satellite cells undergo activation, followed by proliferation. Proliferating satellite cells, which are myogenic precursor cells, eventually exit the cell cycle and fuse with each other to form multinucleated myotubes. Isolated satellite cells from skeletal muscle can be cultured in vitro as satellite cell-derived primary myoblasts $(2,3)$. These primary myoblasts are used for in vitro models of skeletal muscle cell differentiation, self-renewal of satellite cells (4), in vivo satellite cell transplantation (5), and multi-lineage differentiation (6). As opposed to immortalized myoblast cell lines such as $\mathrm{C} 2 \mathrm{C} 12$ cells, animal or human primary myoblasts can be utilized for cell transplantation as well as studies of stem cell biology $(4,7)$.

One drawback of primary myoblasts is that they need more complex culture condi- tions to maintain their proliferation and differentiation abilities. The use of high serum conditions for cell growth is an example of this. Furthermore, the efficiency of DNA transfection and viral infection for primary myoblasts is lower than for $\mathrm{C} 2 \mathrm{C} 12$ cells $(8,9)$. Retroviral or lentiviral infection has been used for obtaining stable foreign gene expression that enables long-term experiments, including in vivo cell transplantation of myogenic cells (2,10-12). However, the viral supernatant normally contains low levels of nutrients and growth factors, which inevitably induces cell cycle exit followed by myogenic differentiation. Therefore, a method for high-efficiency viral infection without the need for culturing with the viral supernatant is critical for maintaining the ability of primary myoblasts to proliferate and differentiate (13).

For efficient retroviral infection, a spin infection protocol has been established for several cell types, including hematopoietic progenitor cells (14-17). To adapt the spin infection method to primary myoblasts, we identified optimal conditions for both transfection reagents and centrifugation time and force.

\section{Materials and methods}

Primary myoblast culture

All animal experimental protocols were approved by Institutional Animal Care and the Use Committee of the University of Minnesota. Satellite cell-derived primary myoblasts such as CD31(-), CD45(-), Sca-1(-), and integrin $\alpha 7(+)$ cells were isolated from skeletal muscles of 2 month-old mice (C57BL6, Charles River Laboratories, Wilmington, MA) by MACS separation (Miltenyi Biotec, San Diego, CA) as described previously (3). Myoblasts were maintained on collagen-coated dishes in growth medium (GM) [Ham's/F10 (SigmaAldrich, St., Louis, MO), 20\% FBS, 20 ng/ $\mathrm{mL}$ basic FGF (R\&D Systems, Minneapolis, $\mathrm{MN}$ ), and 1\% penicillin/streptomycin (Invit-

Here, we compared multiple commercially available transfection reagents with different infection protocols and determined that a spin infection protocol with centrifugation had the highest infection efficiency for primary myoblasts. The infected cells continued to proliferate and retained the capacity for in vitro and in vivo myogenic differentiation. 
rogen, Carlsbad, CA)] (7). Proliferating myoblasts in GM were defined as Day 0. Myogenic differentiation was induced by replacing GM with differentiation medium (DM) [DMEM (Sigma-Aldrich), 5\% horse serum, and 1\% penicillin/streptomycin] for 3 days.

\section{Transfection and viral infection}

Retroviral supernatants were produced by transfection of pMX-GFP (Cell Biolabs, San Diego, CA) or a pMX-mCherry retroviral vector into a 293T Platinum-E Retroviral Packaging Cell Line (Plat-E) (Cell Biolabs). One day before transfection, Plat-E cells were cultured in DMEM with 10\% FBS and without antibiotics until they reached 70\%-90\% confluency. Various transfection reagents were used: Lipofectamine (Thermo Fisher Scientific, Waltham, MA), Lipofectamine 2000 (Thermo Fisher Scientific), Lipofectamine LTX (Thermo Fisher Scientific), TransIT-293 (Mirus Bio LLC, Madison, WI), TransIT-2020 (Mirus Bio LLC), TransIT-LT1 (Mirus Bio LLC), PolyJet (SignaGen Laboratories, Rockville, MD), and LipoJet (SignaGen Laboratories). Five microliters of each transfection reagent was suspended in $200 \mu \mathrm{l}$ DMEM without FBS and with $5 \mu \mathrm{g}$ of pMX-GFP or pMX-mCherry plasmid DNA for 20 min at room temperature (RT). PlatE cells (6 $\times 10^{5}$ ) were plated on collagen-coated $3 \mathrm{~cm}$ dishes 1 day before transfection. The next day, the medium was replaced with $800 \mu \mathrm{l}$ DMEM with 10\% FBS and $200 \mu$ I DMEM using the transfection complex described above. After incubation for $24 \mathrm{~h}$, the medium was changed to $1 \mathrm{~mL}$ new DMEM with 10\% FBS. Retroviral supernatants were then harvested $24 \mathrm{~h}$ after the medium change. Syringe filters $(0.45 \mu \mathrm{m})$ (Millipore Sigma, Billerica, MA) were used to remove any cells from the retroviral supernatants. Primary myoblasts $(1 \times$ $10^{5}$ ) were plated on collagen-coated $3 \mathrm{~cm}$ dishes for $24 \mathrm{~h}$ before the viral plating infection. Retroviral supernatants were used for viral infection of primary myoblasts with $10 \mu \mathrm{g} / \mathrm{mL}$ polybrene (Millipore Sigma) for $4 \mathrm{~h}$, and cells were then cultured in GM for $48 \mathrm{~h}$. For the spin infection, myoblasts were treated with $0.25 \%$ trypsin-EDTA (Thermo Fisher Scientific), and $1 \times 10^{5}$ myoblasts were then transferred into $1.5 \mathrm{~mL}$ microcentrifuge tubes. The cells were centrifuged and then resuspended with the retroviral supernatant with $10 \mu \mathrm{g} /$ $\mathrm{mL}$ polybrene. After the myoblast spin infection was performed at RT under appropriate centrifugation conditions, the cell pellets were resuspended with $\mathrm{GM}$ and plated on collagen-coated $3 \mathrm{~cm}$ dishes. GFP expression was examined 2 days after spin infection. Dead cells were counted by trypan blue (Thermo Fisher Scientific) staining. 5-ethynyl-2'-deoxyuridine (EdU) was added to culture plates $3 \mathrm{~h}$ before fixation of the cells. EdU staining was performed using the Click-iT EdU Alexa Fluor 488 Imaging Kit (Thermo Fisher Scientific). Immunostaining was performed with anti-GFP (AB3080; Millipore Sigma; RRID:AB_91337), anti-myosin heavy chain (MHC) (MF 20; Developmental Study Hybridoma Bank, lowa City, IA; RRID:AB_2147781), anti-myogenin antibody (F5D; Developmental Study Hybridoma Bank; RRID:AB_2146602), or anti-phosphohistone H3 antibody (pHisH3) (D2C8; Cell Signaling, Danvers, MA; RRID:AB_10694226), followed by Alexa 488-conjugated anti-rabbit IgG (A-21206; Thermo Fisher Scientific; RRID:AB_2535792) and Alexa 568-conjugated anti-mouse IgG (A10037; Thermo Fisher Scientific; RRID:AB_2534013) or Alexa 488-conjugated anti-mouse IgG (A-21202; Thermo Fisher Scientific; RRID:AB_141607). DAPI (Sigma-Aldrich) was used for counterstaining of nuclei.

Intramuscular injection of myoblasts

All animals were maintained inside a barrier facility, and all in vivo experiments were performed in accordance with the guidelines of
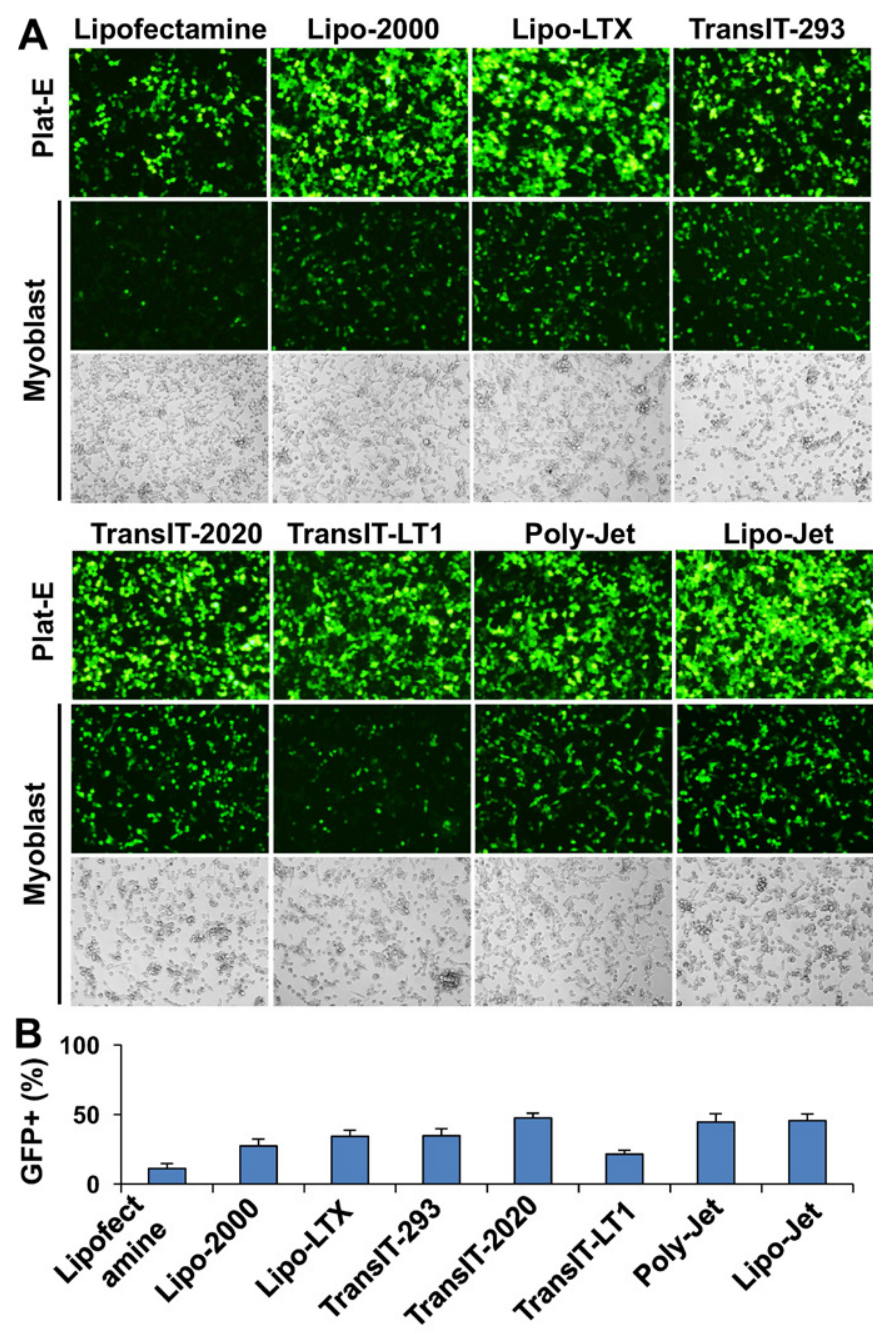

Figure 1. GFP expression after transfection of Plat-E cells and infection of primary myoblasts. Transfection reagents Lipofectamine 2000, Lipofectamine LTX, TransIT-293, TransIT-2020, TransIT-LT1, PolyJet, or LipoJet were used for pMX-GFP retroviral vector transfection. (A) GFP expression in Plat-E cells 2 days after transfection using each transfection reagent, and after infection of primary myoblasts with indicated viral supernatants for 2 days. These phase contrast images show primary myoblasts after infection. (B) The percentage of GFP-positive primary myoblasts out of total cells after infection. Data are presented as mean $\pm \operatorname{SEM}(n=3)$.

the Institutional Animal Care and Use Committee of the University of Minnesota for the care and use of laboratory animals. pMXs- or pMX-mCherry-infected myoblasts $\left(1 \times 10^{6}\right)$ were used for engraftment in one tibialis anterior (TA) muscle of a Nod:Scid immunodeficient mouse (Charles River Laboratories). PBS injection was performed as the control. Two days before myoblast injection, $50 \mu \mathrm{l} 2.5 \mu \mathrm{M}$ cardiotoxin (CTX) (Sigma-Aldrich) was intramuscularly injected into the TA muscle to induce muscle injury. The TA muscle was harvested for histological analysis 1 or 3 months after cell injection. Frozen sections $(8 \mu \mathrm{m})$ were prepared for engraftment analysis. Anti-laminin antibody (L0663; Sigma-Aldrich; RRID:AB_477153) followed by Alexa 488-conjugated anti-rat IgG (A-21208; Thermo Fisher Scientific; RRID:AB_141709) and DAPI (Sigma-Aldrich) were used for immunostaining. Hematoxylin and eosin (HE) staining and Sirius red staining (Sigma-Aldrich) were also performed 1 month after cell injection. At that time, myofibers were isolated from mouse extensor digitorum longus (EDL). Muscles were incubated in DMEM with $0.2 \%$ collagenase type I (Sigma-Aldrich) at $37^{\circ} \mathrm{C}$ for $2 \mathrm{~h}$, trans- 
A

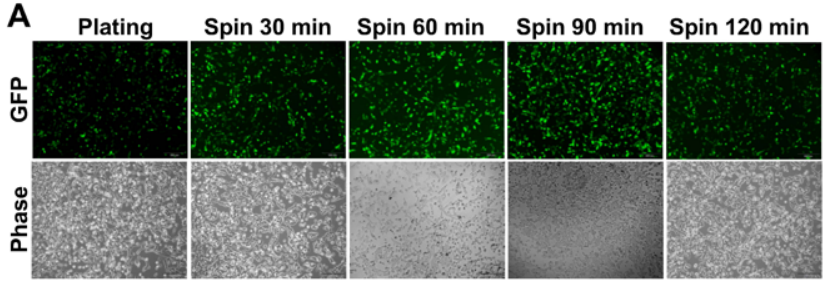

B

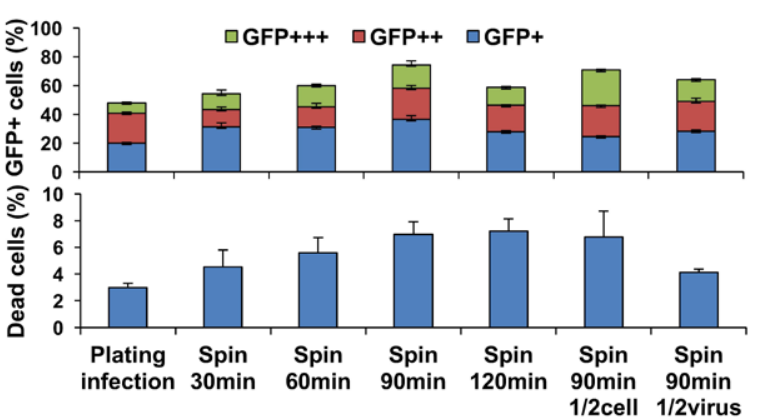

C
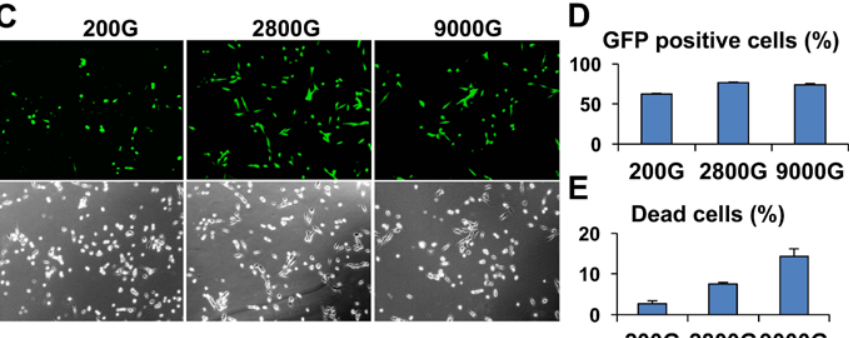

Figure 2. Comparison of retroviral infection efficiencies by spin infection and plating infection. (A) GFP-positive primary myoblasts 2 days after plating infection or spin infection (upper panels) and their phasecontrast images (lower panels). (B) The percentage of GFP-positive primary myoblasts 2 days after infection. GFP+++, ++ , and + denote expression levels GFPhigh, GFPmed, and GFPlow, respectively. The percentage of trypan blue-positive dead primary myoblasts 2 days after infection is shown below. Data are presented as the mean \pm SEM $(n=$ 3). (C) GFP-positive primary myoblasts 2 days after spin infection (upper panels) and their phase-contrast images (lower panels). (D) The percentage of GFP-positive primary myoblasts 2 days after infection. (E) The percentage of trypan blue-positive dead primary myoblasts 2 days after infection. Data are presented as the mean $\pm \operatorname{SEM}(n=3)$.

ferred into DMEM, and triturated to release single myofibers. Single muscle fibers were immunostained with anti-Pax7 antibody (Pax7; Developmental Study Hybridoma Bank; RRID:AB_528428) followed by Alexa 488-conjugated anti-mouse IgG (A-21202; Thermo Fisher Scientific; RRID:AB_141607). DAPI was used for counterstaining of nuclei.

\section{Statistics}

Data are presented as mean \pm SEM. Statistical comparisons were performed using an unpaired two-tailed Student's $t$ test or ANOVA with Scheffe's test with $P<0.05$ as the threshold for statistical significance.

\section{Results and discussion}

Transfection reagents for retroviral infection of primary myoblasts

For retroviral infection, a high titer is important for efficient expression of the ectopic gene. We first examined several commercially available transfection reagents for transfection into Plat-E virus packaging cells, which are superior to 293T cells. We transfected cells with a pMX-GFP plasmid vector using Lipofectamine, Lipofectamine 2000,
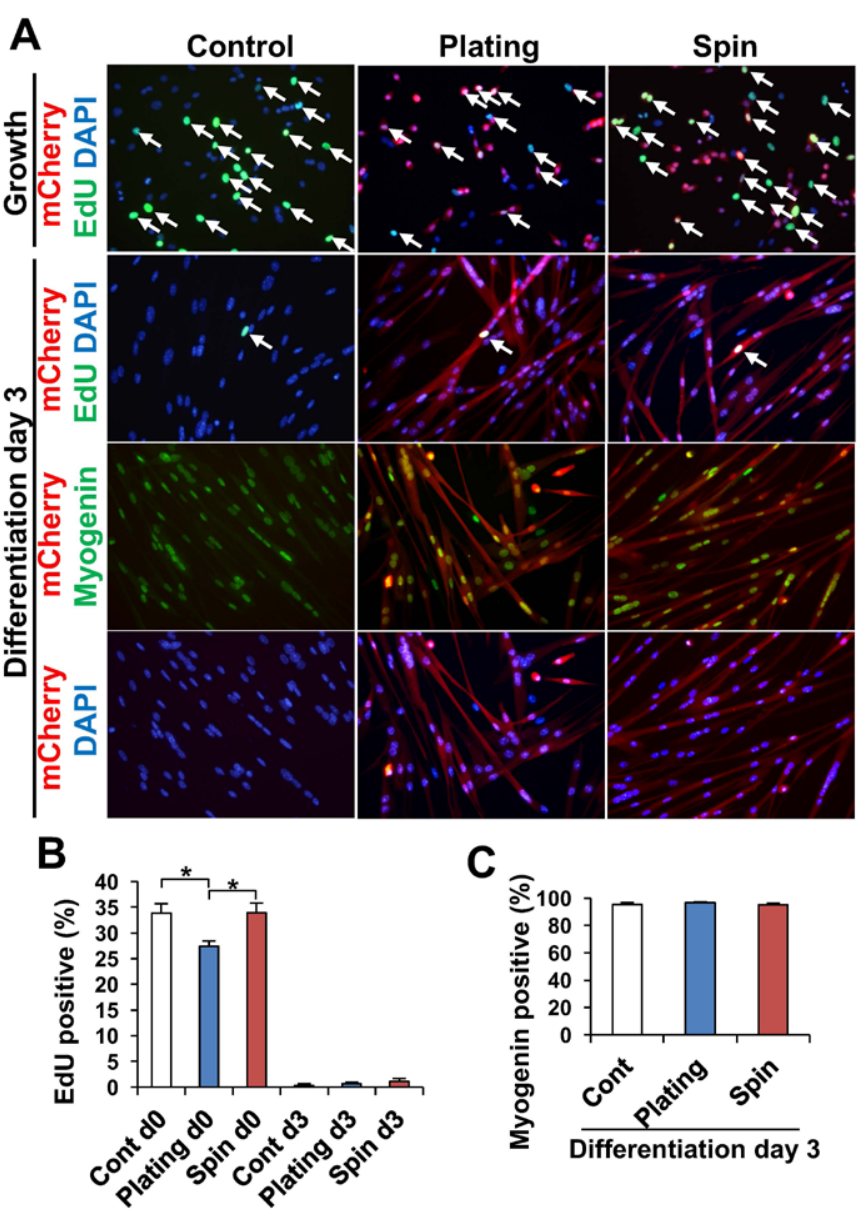

Figure 3. Cell proliferation and myogenic differentiation of primary myoblasts after retroviral spin cell infection. (A) mCherry-positive primary myoblasts following plating or spin infection were analyzed by EdU incorporation or myogenin staining (d0: growth, d3: differentiation day 3). Control primary myoblasts contained no virus-infected cells. $(B, C)$ The percentage of EdU- or myogenin-positive primary myoblasts. DAPI was used to counterstain nuclei. Control denotes no virus-infected cells. Asterisks indicate statistically significant differences $\left({ }^{*}: P<0.05\right)$. Data are presented as the mean $\pm \operatorname{SEM}(n=3)$.

Lipofectamine LTX, TransIT-293, TransIT-2020, TransIT-LT1, PolyJet, or LipoJet. Two days after transfection, all transfection reagents allowed Plat-E cells to express GFP (Figure 1A). Lipofectamine and TransIT-293 showed lower transfection efficiencies than the other reagents. After plating infection of primary myoblasts with these retroviral supernatants, the infection efficiencies quantified by GFP-positive cells were quite different. TransIT-2020 (47.33 $\pm 3.62 \%)$, PolyJet (44.32 \pm $6.12 \%)$, and LipoJet ( $45.53 \pm 4.76 \%)$ displayed relatively higher infection efficiencies compared to the other reagents (Figure 1, A and B). Among those three reagents, we chose PolyJet as the transfection reagent for subsequent spin infection experiments due to its cost effectiveness. We also compared 2 different ratios of the amounts of pMX-GFP DNA to PolyJet, 1:1 and 1:2, for the transfection step and found no significant difference in transfection efficiency (Supplementary Figure S1).

Spin infection of primary myoblasts

We tested infection of primary myoblasts using retroviral supernatants by spin infection at $2800 \times g$ for different time periods (30, 60, 90 and $120 \mathrm{~min}$ ). First, we trypsinized primary myoblasts to obtain small cell pellets, enabling cells to be incubated with a small volume of retroviral supernatant. Two days after plating, spin 


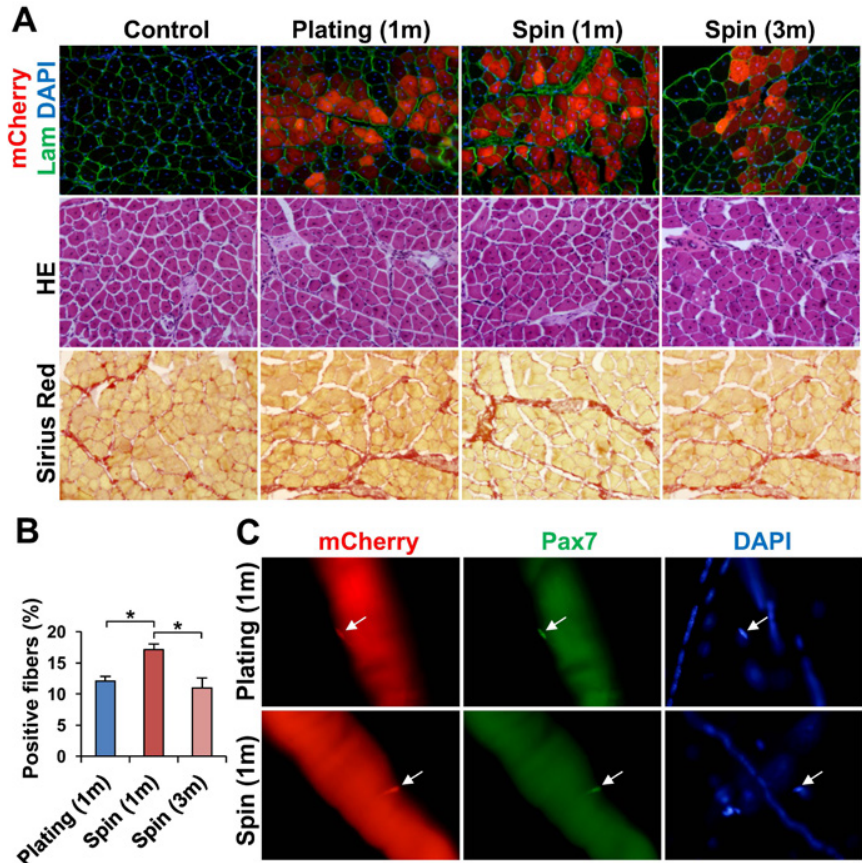

Figure 4. In vivo myogenic differentiation of primary myoblasts after plating and spin infection. (A) mCherry-positive primary myoblasts following plating or spin infection were intramuscularly injected into regenerating tibialis anterior (TA) muscle 2 days after cardiotoxin (CTX) injection. TA muscle was harvested 1 or 3 months after cell injection. mCherry and laminin (Lam)-positive muscle fibers were detected as an engraftment. Hematoxylin and eosin (HE) and Sirius red staining display the muscle histology 1 month after cell injection. PBS injection was used for the control muscle. (B) Percentages of mCherry-positive muscle fibers for each method. (C) mCherry-positive donor-derived satellite cells (red) expressed Pax7 (green) in isolated single myofibers. DAPI was used for counterstaining of nuclei. Asterisks indicate statistically significant differences ( $\left.{ }^{*}: P<0.05\right)$. Data are presented as mean $\pm \operatorname{SEM}(n=3)$.

infection for 90 min showed the highest efficiency $(74.40 \pm 6.93 \%)$. Importantly, the efficiency of this spin infection methodology was significantly better than the efficiency of a classical plating infection method (47.94 $\pm 4.39 \%$ ) (Figure 2, A and B). When a lower retroviral supernatant concentration was used, the infection efficiency was reduced (63.93 $\pm 6.45 \%)$ (Figure $2 \mathrm{~B})$. When using half of the number of primary myoblasts, cells highly expressing GFP (GFPhigh) were increased $(24.91 \pm 0.53 \%)$ compared with all of the cells (16.22 $\pm 2.72 \%$ ) (Figure $2 \mathrm{~B}$ ), indicating that each primary myoblast was infected with more copies of the retrovirus. We also counted dead cells using trypan blue staining. The number of dead cells was increased when higher relative centrifugal forces (RCFs) were used for spin infection. However, the dead cells in each condition were still less than $10 \%$ of the total primary myoblasts (Figure 2B).

Next, we tested spin infection conditions with 3 different RCFs $(200,2800$, and $9000 \times g)$ for $90 \mathrm{~min}$. Two days after spin infection, we found that the $2800 \times g$ RCF showed the highest efficiency (76.94 $\pm 2.27 \%$ ) (Figure 3, C and D). Trypan blue staining showed that cell death was increased as RCF increased (Figure 3E). Taking this into account, we concluded that spin infection at $2800 \times g$ for $90 \mathrm{~min}$ is the most effective method for infecting primary myoblasts.

Characterization of infected primary myoblasts

Compared with myoblast cell lines such as $\mathrm{C} 2 \mathrm{C} 12$ cells, primary myoblasts are less stable and lose their myogenic differentiation capability easily. Following spin infection at $2800 \times g$ for 90 min, we noticed that the number of EdU-positive or pHisH3-positive proliferating primary myoblasts was increased, while the number of myosin heavy chain (MHC)-positive differentiating myocytes was decreased compared with adherent (plating) infection (Figure 3, A and B; Supplementary Figure S2, A and B). We confirmed, however, that myogenin-positive myotube formation by the primary myoblasts was still maintained following spin infection at $2800 \times$ $g$ for 90 min (Figure 3, A and C). Next, following plating or spin infection at $2800 \times g$ for 90 min, we attempted intramuscular transplantation of the infected primary myoblasts into cardiotoxin (CTX)induced regenerating TA muscle of Nod:Scid immunodeficient mice. One month after cell injection, more mCherry-positive muscle fibers were detected after injection with spin-infected primary myoblasts compared with plating-infected primary myoblasts. HE and Sirius red staining showed no differences in histological morphology and fibrosis between regenerating muscle injected with PBS, platinginfected primary myoblasts, or spin-infected primary myoblasts. Three months after cell injection, mCherry-positive muscle fibers were still detected (Figure 4, A and B). In both injected groups, Pax7-positive satellite cells were detected in isolated single myofibers from the recipient mice (Figure $4 \mathrm{C}$ ). These results indicate that spininfected primary myoblasts display better engraftment and myogenic differentiation following intramuscular injection.

Here, we demonstrated high-efficiency retroviral infection into primary myoblasts using spin infection with high-speed centrifugation. These spin-infected primary myoblasts showed better cell proliferation in GM and underwent proper myogenic differentiation in DM. Furthermore, these spin-infected primary myoblasts could engraft into regenerating muscle fibers after intramuscular injection more efficiently than plating-infected myoblasts or Pax7-positive satellite cells. We also identified effective transfection reagents for the production of retroviral supernatant used for infection of primary myoblasts. High efficiency of retroviral infection is crucial for primary myoblasts since primary myoblasts are a heterogeneous population (4); therefore, selective infections may not be representative of the overall population. Unlike myoblast cell lines such as C2C12 cells, primary myoblasts sometimes lose their myogenic differentiation capacity. However, our data clearly showed that the spin infection method does not affect the myogenic differentiation capability of primary myoblasts, meaning it is suitable for use in regenerative medicine.

Previous experiments using spin infection with adherent cells were performed using multi-well plates with retroviral supernatants (9). However, our spin infection method was performed in microcentrifuge tubes with small cell pellets after trypsinizing cells from the plates, in the same way as non-adherent cells. This new method has advantages since relatively large numbers of cells can be infected with a reduced volume of retroviral supernatants and, thus, lower amounts of viral vector DNA, transfection reagents, and viral producing cells are needed. Taken together, our spin infection method can enable efficient viral vector-mediated gene expression, not only in primary myoblasts but also in many adherent and non-adherent cell types.

\section{Author contributions}

Y.K. and Y.A. performed the experiments, gathered data, and wrote the manuscript. A.A. created the figures and wrote the manuscript. 


\section{Acknowledgments}

We thank Conor Burke-Smith for critical reading. This work was supported by the NIH R01 (1R01AR062142) and NIH R21 (1R21AR070319). This paper is subject to the NIH Public Access Policy.

\section{Competing interests}

The authors declare no competing interests.

\section{References}

1. Mauro, A. 1961. Satellite cell of skeletal muscle fibers. J. Biophys. Biochem. Cytol. 9:493-495.

2. Rando, T.A. and H.M. Blau. 1994. Primary mouse myoblast purification, characterization, and transplantation for cell-mediated gene therapy. J. Cell Biol. 125:12751287.

3. Motohashi, N., Y. Asakura, and A. Asakura. 2014. Isolation, culture, and transplantation of muscle satellite cells. J. Vis. Exp._86:50846.

4. Sacco, A., R. Doyonnas, P. Kraft, S. Vitorovic, and H.M. Blau. 2008. Self-renewal and expansion of single transplanted muscle stem cells. Nature 456:502-506.

5. Montarras, D., J. Morgan, C. Collins, F. Relaix, S. Zaffran, A. Cumano, T. Partridge, and M. Buckingham. 2005. Direct isolation of satellite cells for skeletal muscle regeneration. Science 309:2064-2067.

6. Asakura, A., M. Komaki, and M. Rudnicki. 2001. Muscle satellite cells are multipotential stem cells that exhibit myogenic, osteogenic, and adipogenic differentiation. Differentiation 68:245-253.

7. Asakura, A., P. Seale, A. Girgis-Gabardo, and M.A. Rudnicki. 2002. Myogenic specification of side population cells in skeletal muscle. J. Cell Biol. 159:123-134.

8. Dhawan, J., L.C. Pan, G.K. Pavlath, M.A. Travis, A.M. Lanctot, and H.M. Blau. 1991. Systemic delivery of human growth hormone by injection of genetically engineered myoblasts. Science 254:1509-1512.
9. Kodaka, Y., K. Tanaka, K. Kitajima, K. Tanegashima, R. Matsuda, and T. Hara. 2015. LIM homeobox transcription factor Lhx2 inhibits skeletal muscle differentiation in part via transcriptional activation of Msx1 and Msx2. Exp. Cell Res. 331:309-319.

10. Smith, B.F., R.K. Hoffman, U. Giger, and J.H. Wolfe. 1990. Genes transferred by retroviral vectors into normal and mutant myoblasts in primary cultures are expressed in myotubes. Mol. Cell. Biol. 10:3268-3271.

11. Yao, S.N. and K. Kurachi. 1992. Expression of human factor IX in mice after injection of genetically modified myoblasts. Proc. Natl. Acad. Sci. USA 89:33573361.

12. Huard, J., G. Acsadi, A. Jani, B. Massie, and G. Karpati. 1994. Gene transfer into skeletal muscles by isogenic myoblasts. Hum. Gene Ther. 5:949-958.

13. Jackson, M.F., K.E. Hoversten, J.M. Powers, G.D. Trobridge, and B.D. Rodgers. 2013. Genetic manipulation of myoblasts and a novel primary myosatellite cell culture system: comparing and optimizing approaches. FEBS J. 280:827-839.

14. Kotani, H., P.B. Newton 3rd, S. Zhang, Y.L. Chiang, E. Otto, L. Weaver, R.M. Blaese, W.F. Anderson, and G.J. McGarrity. 1994. Improved methods of retroviral vector transduction and production for gene therapy. Hum. Gene Ther. 5:19-28.

15. Pear, W. 2001. Transient transfection methods for preparation of high-titer retroviral supernatants. Curr Protoc Mol Biol Chapter 9:Unit9 11.

16. Biagi, E., F. Bambacioni, G. Gaipa, C. Casati, J. Golay, A. Biondi, and M. Introna. 2001. Efficient lentiviral transduction of primary human acute myelogenous and lymphoblastic leukemia cells. Haematologica 86:13-16.

17. Tong, H.I., W. Kang, G. Zhou, M. Liu, Y. Shi, and Y. Lu. 2016. Evaluation on monocyte-mediated delivery of a therapeutic gene into the inflamed brain. Curr. Gene Ther. 16:401-409.

Received 24 June 2016; accepted 28 June 2017

Address correspondence to Atsushi Asakura, Stem Cell Institute, University of Minnesota, McGuire Translational Research Facility, Room 4-220, 2001 6th Street SE, Mail Code 2873, Minneapolis, Minnesota, 55455. E-mail: asakura@umn.edu

To purchase reprints of this article, contact:

biotechniques@fosterprinting.com

\section{THE HIGHEST YIELD AND PURITY:}

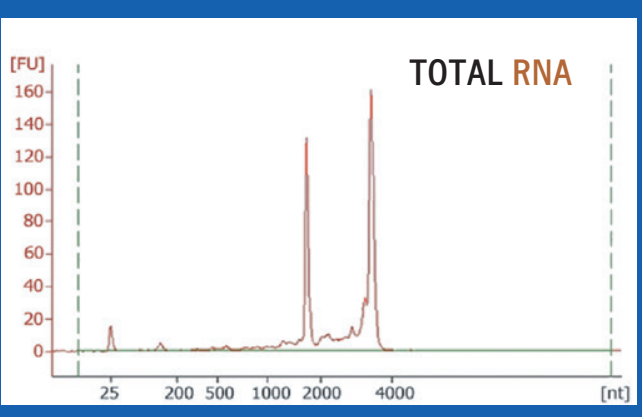

The single-step method without phase separation

$\checkmark$ No DNase treatment necessary

$\checkmark$ RNA ready for RT-PCR, microarrays and other applications

No need for refrigerated centrifuge

One reagent for solid and liquid samples

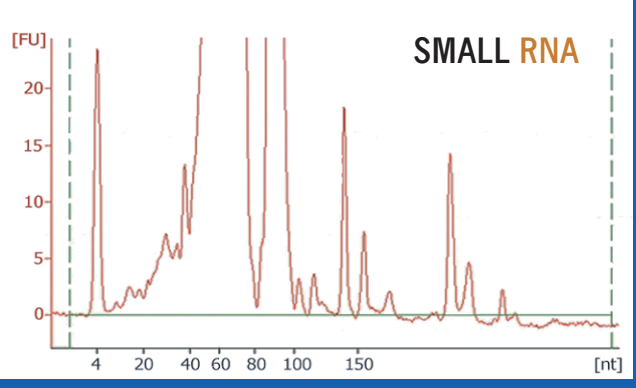

